\title{
Commentary
}

Neonatology

Neonatology

DOI: 10.1159/000499742
Received: March 6, 2019

Accepted after revision: March 19, 2019

Published online: June 11, 2019

\section{Phenobarbital Increases Midazolam Clearance in Neonates Treated with Hypothermia: Do We Really Need to Know?}

\author{
Karel Allegaert ${ }^{a, b}$ Anne Smits ${ }^{b, c}$ John N. van den Anker ${ }^{d-f}$ \\ a Division of Neonatology, Department of Pediatrics, Sophia Children's Hospital, Erasmus MC, Rotterdam, \\ The Netherlands; ${ }^{\mathrm{b}}$ Neonatal Intensive Care Unit, Department of Development and Regeneration, KU Leuven, \\ Leuven, Belgium; ' $N e o n a t a l$ Intensive Care Unit, University Hospitals Leuven, Leuven, Belgium; ${ }^{\mathrm{d}}$ Department of \\ Pediatric Surgery, Sophia Children's Hospital, Erasmus MC, Rotterdam, The Netherlands; ${ }^{\circ}$ Division of Clinical \\ Pharmacology, Department of Pediatrics, Children's National Health System, Washington, DC, USA; ${ }^{\mathrm{f} D i v i s i o n}$ of \\ Paediatric Pharmacology and Pharmacometrics, University of Basel Children's Hospital, Basel, Switzerland
}

The clinical management and subsequent outcome of pediatric and neonatal patients can improve significantly with the availability of effective and safe medicines if appropriately investigated in the relevant population [1]. This is also the case for neonates treated with hypothermia for perinatal asphyxia. However, the vast majority of medicines are developed with adult pathophysiology in mind and are not guided by neonatal (patho)physiology. Drug development is mainly driven by adult indications, subsequently tailored or repurposed for use in neonates, with exogenous surfactant as the latest but hopefully not last example of drug discovery specific to neonates [2].

Since there is level I evidence in support of therapeutic hypothermia for asphyxiated neonates (number needed to treat: $7,95 \%$ CI 5-10), there is a very active research line investigating add-on pharmacotherapy to further improve this outcome $[2,3]$. After an unstructured search on the European Medicines Agency (EMA) website, allopurinol, Argon, Xenon, VH-N439, cannabidiol, 2-iminobiotin, melatonin, and erythropoietin development plans were retrieved (besides stem cell-related approaches) as orphan development programs for this indication. In the setting of an orphan indication, with the need for immediate neonatal intervention and, as a consequence,

\section{KARGER}

๑ 2019 S. Karger AG, Basel

E-Mail karger@karger.com

www.karger.com/neo a very high logistic burden to conduct this kind of studies, we need to generate as much as possible add-on knowledge from the currently available fragmented data on (patho)physiological changes in organ function and blood flow, or drug-specific pharmacokinetics to make these studies more feasible and explore the underlying mechanisms in this specific clinical setting.

Using an opportunistic sampling approach and nonlinear mixed effects modeling techniques [4], Favié et al. [5] quantified the impact of phenobarbital co-administration on midazolam clearance (factor 2.3 higher, 95\% CI 1.9-2.9) in neonates undergoing therapeutic hypothermia, while the subsequent $1^{\prime}$-hydroxymidazolam clearance (glucuronidation and renal elimination) was reduced $(-25 \%)$ by hypothermia. Based on our experience and expertise in perinatal pharmacology, we would like to draw the attention of clinicians and clinician scientists to the relevance of this new information for clinical management and neonatal drug development.

As mentioned by the authors, the consequence of these findings is that midazolam clearance in neonates is already driven to a clinically relevant extent by phenobarbital co-exposure, indicating the capacity of this frequently used drug to induce cytochrome P450 (CYP)3A 


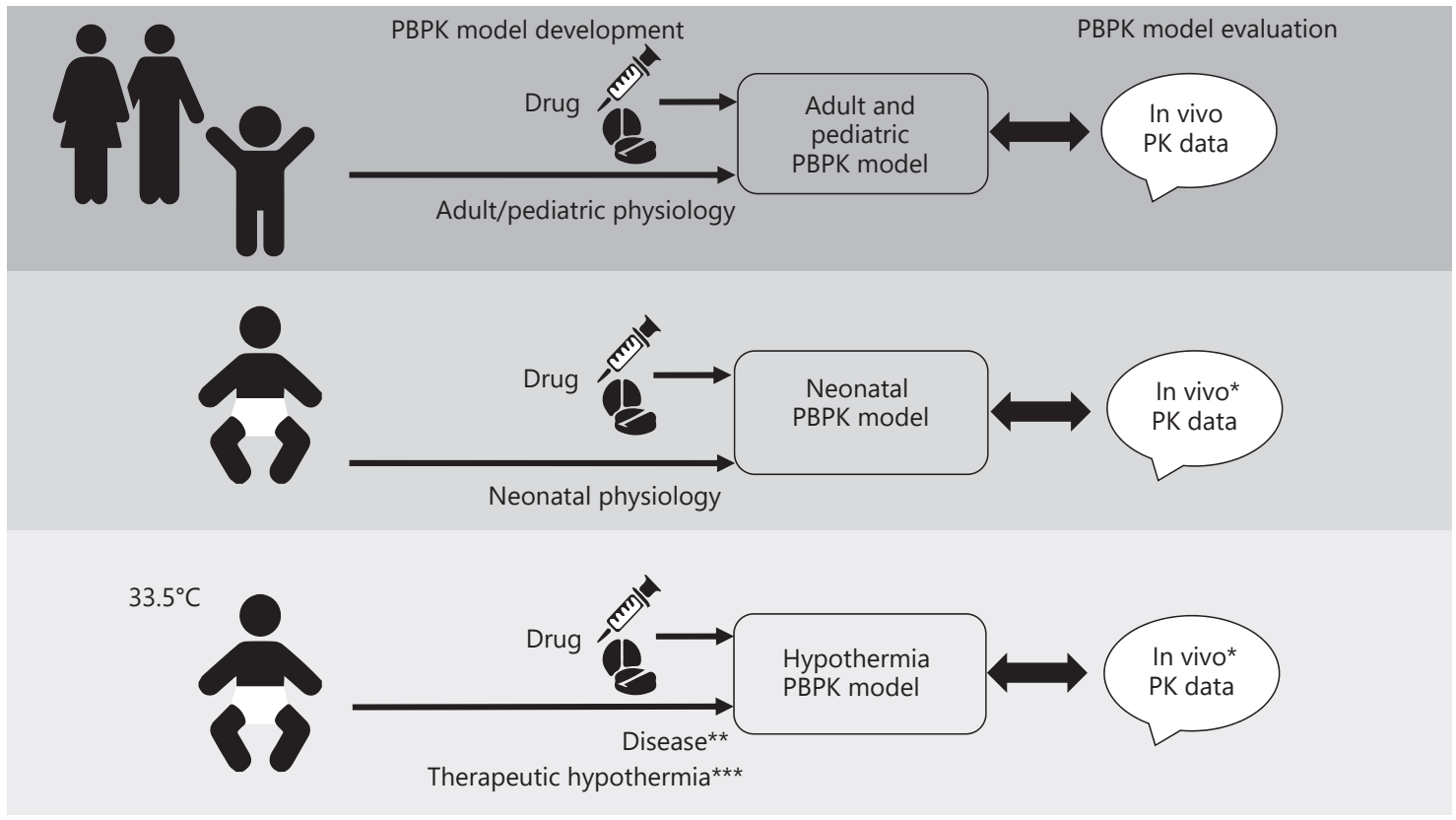

Fig. 1. Development and validation of PBPK models, specific to neonates undergoing therapeutic hypothermia. Such a workflow necessitates - besides drug specific physicochemical input - data sharing and availability of in vivo PK data in the population of interest (neonates, ${ }^{*}$ ), but also data on the disease state (asphyxia, ${ }^{* *}$ ) and on the impact of hypothermia $(* * *)$.

activity already early in life. Similar patterns can be anticipated for other drugs when co-administered with phenobarbital, such as sildenafil (co-occurrence pulmonary hypertension) or fentanyl (narcotic) [6,7]. For all these drugs, a fast postnatal age-driven maturational increase in clearance is described, and it is reasonable to anticipate that this pattern will be further enhanced when phenobarbital is co-administered [6, 7].

The other way around, clinicians should also be aware that shifts in antiepileptic drug (AED) prescription practices may also result in additional effects because of shifts in drug utilization may result in shifts in the occurrence of drug-drug interactions. Using the Pediatrix database on medication use in neonates, Ahmad et al. [8] reported that neonates with seizures are still almost all exposed to phenobarbital with a decrease (15-11\%) in the use of phenytoin mirrored by a significant increase $(1.4-14 \%)$ in levetiracetam prescription over time (2005 to 2014) [9]. Some authors advocate the use of other AEDs like levetiracetam as first-line AED in neonates $[5,10]$. If so, a similar dosing $(\mathrm{mg} / \mathrm{kg})$ of benzodiazepines as second-line AED will result in higher exposure to benzodiazepines in levetiracetam cases because of the absence of phenobarbital-related induction.
Besides the clinical relevance, this detailed PK analysis also unveiled changes in metabolic and elimination pathways, and such information is important beyond drugspecific observations: neonatal pharmacology reflects developmental (patho)physiology [11]. Variability is the core business of neonatal pharmacology because development and growth (weight gain) are most prominent in early infancy, while PK are further affected by nonmaturational covariates such as polymorphisms or environmental (drug-drug, drug-nutrition, drug-treatment modalities, disease, but also therapeutic hypothermia + perinatal asphyxia) factors $[2,11]$.

Physiologically based PK (PBPK) techniques provide a potent systematic approach to make the most of already acquired knowledge (physiology, system knowledge) to capture the variability, to adapt dosing, or to assist in the trial design in neonates $[12,13]$. PBPK hereby integrates different types of information, such as clinical data and in silico, in vitro, and in vivo observations to predict drug exposure over time. PBPK hereby explicitly discriminates between population physiological properties (system parameters such as cardiac output, organ perfusion or blood flow, renal function, liver size, weight, plasma protein, different between and within populations) and drug-spe- 
cific (chemical, $\mathrm{pH}$, solubility) properties, not different between populations (Fig. 1).

Progress in this field, however, necessitates contributions of clinicians by generating datasets on PK and maturational physiology to use these datasets to refine PBPK model predictions. This necessitates data sharing and availability of in vivo $\mathrm{PK}$ data in the population of interest (newborns, ${ }^{*}$ ), data on disease state (asphyxia, ${ }^{* *}$ ), and the impact of hypothermia itself $(* * *)$, and this is exactly why clinicians should become aware of the relevance of such data beyond drug-specific relevance. Once developed and validated, such PBPK tools may indeed be instrumental to assure that studies on pharmacological interventions become much more feasible. Using this approach, it has applications in first-in-adult/child, first-in-newborn, or first-in the-newborn-treated-with-hypothermia drug development. The final intention is to generate dosing recommendations, or alternatively, simulations to subsequently conduct PK studies, also in this specific hypothermia setting.

\section{References}

1 van den Anker J, Reed MD, Allegaert K, Kearns GL. Developmental changes in pharmacokinetics and pharmacodynamics. J Clin Pharmacol. 2018 Oct;58 Suppl 10:S1025.

2 Allegaert K, van den Anker JN. Clinical pharmacology in neonates: small size, huge variability. Neonatology. 2014;105(4):344-9.

3 Jacobs SE, Berg M, Hunt R, Tarnow-Mordi WO, Inder TE, Davis PG. Cooling for newborns with hypoxic ischaemic encephalopathy. Cochrane Database Sys Rev. 2013 Jan;(1):CD003311.

4 De Cock RF, Piana C, Krekels EH, Danhof M, Allegaert K, Knibbe CA. The role of population PK-PD modelling in paediatric clinical research. Eur J Clin Pharmacol. 2011 May; 67(S1 Suppl 1):5-16.
5 Favié LMA, Groenendaal F, van den Broek MPH, Rademaker CMA, de Haan TR, van Straaten HLM, et al; on behalf of the PharmaCool study group. Phenobarbital, midazolam pharmacokinetics, effectiveness, and drugdrug interaction in asphyxiated neonates undergoing therapeutic hypothermia. Neonatology. DOI: 10.1159/000499330.

6 Mukherjee A, Dombi T, Wittke B, Lalonde R. Population pharmacokinetics of sildenafil in term neonates: evidence of rapid maturation of metabolic clearance in the early postnatal period. Clin Pharmacol Ther. 2009 Jan;85(1): 56-63.

7 Völler S, Flint RB, Andriessen P, Allegaert K, Zimmermann LJ, Liem KD, et al. Rapidly maturing fentanyl clearance in preterm neonates. Arch Dis Child Fetal Neonatal Ed. 2019 Feb. Epub 2019 Feb 1. DOI: 10.1136/archdischild-2018-315920.

8 Ahmad KA, Desai SJ, Bennett MM, Ahmad SF, Ng YT, Clark RH, et al. Changing antiepileptic drug use for seizures in US neonatal intensive care units from 2005 to 2014. J Perinatol. 2017 Mar;37(3):296-300.
9 Allegaert K, Simons S, Van Den Anker J. Research on medication use in the neonatal intensive care unit. Expert Rev Clin Pharmacol. 2019 Apr;12(4):343-53.

10 El-Dib M, Soul JS. The use of phenobarbital and other anti-seizure drugs in newborns. Semin Fetal Neonatal Med. 2017 Oct;22(5):3217.

11 Krekels EH, van Hasselt JG, van den Anker JN, Allegaert K, Tibboel D, Knibbe CA. Evidence-based drug treatment for special patient populations through model-based approaches. Eur J Pharm Sci. 2017 Nov; 109S:S22-6.

12 Smits A, De Cock P, Vermeulen A, Allegaert K. Physiologically based pharmacokinetic (PBPK) modeling and simulation in neonatal drug development: how clinicians can contribute. Expert Opin Drug Metab Toxicol. 2018 Dec;1-10.

13 Michelet R, Bocxlaer JV, Vermeulen A. PBPK in preterm and term neonates: a review. Curr Pharm Des. 2017;23(38):5943-54. 